\title{
Diagnostic, therapeutic and prognostic aspects of uterine synechiae managed by hysteroscopy in the gyanecological endoscopic surgery and human reproductive teaching hospital
}

\section{Etienne Belinga $^{1,2 *}$, Isidore Tompeen ${ }^{2}$, Claude Cyrille Noa Ndoua ${ }^{1,2}$, Junie Metogo Ntsama ${ }^{1,2}$, Sandrine Mendibi ${ }^{1}$, Jean Marie Kasia ${ }^{1,2}$}

\author{
${ }^{1}$ The Gynecological Endoscopic Surgery and Human Reproductive Teaching Hospital, Yaounde, Cameroon \\ (CHRACERH) \\ ${ }^{2}$ Department of Obstetrics and Gynaecology, Faculty of Medicine and Biomedical Sciences, The University of \\ Yaounde I, Cameroon
}

Received: 11 November 2017

Accepted: 09 December 2017

\section{*Correspondence: \\ Dr. Etienne Belinga, \\ E-mail: belingaetienne@yahoo.fr}

Copyright: ( $)$ the author(s), publisher and licensee Medip Academy. This is an open-access article distributed under the terms of the Creative Commons Attribution Non-Commercial License, which permits unrestricted non-commercial use, distribution, and reproduction in any medium, provided the original work is properly cited.

\begin{abstract}
Background: Uterine synechiae refers to a total or partial union of the inner walls of the uterus resulting from endometrial trauma. It is a cause of reproductive failure. Until now, synechia was not optimally treated and has remained understudied in Cameroon. Objective of present study was to Evaluate the diagnostic, therapeutic and prognostic aspects of uterine synechiae treated by hysteroscopy.

Methods: We conducted a descriptive cross-sectional study with an analytical component from January 1 st 2015 to July 31st 2017 at Yaounde. All patients diagnosed with uterine synechiae and treated with hysteroscopy were our sample. Sampling was consecutive. The chi-square test was used for the comparison of qualitative variables and Fisher's test for ANOVA variance analysis. The comparison of the averages was made by the Student's test. P-value less than 0.05 was considered as statistically significant.

Results: Hysteroscopies were indicated for uterine synechiae in $14.50 \%$. Nulliparous were $56.67 \%$. A history of curettage/aspiration was present in $66.66 \%$. All of the patients had a form of infertility and $83.33 \%$ had menstrual disorders. Hysterosalpingography showed a better sensitivity (88\%). After hysteroscopic treatment, $63.30 \%$ had a complete anatomical restitution. There is a significant correlation between the stage of severity of synechia and anatomical restitution ( $\mathrm{p}=0.008$; Spearman correlation coefficient=-0.477).

Conclusions: Uterine synechiae represent one-sixth of all indications for hysteroscopy and present clinically as menstrual disorder associated with infertility. A past history of uterine curettage is common. Hysterosalpingography has a better preoperative diagnostic sensitivity. Hysteroscopy allows optimal treatment.
\end{abstract}

Keywords: CHRACERH, Cameroon, Operative hysteroscopy, Prognosis, Uterine synechiae

\section{INTRODUCTION}

Uterine synechiae are a partial or complete union of the inner walls of the uterus, as a result of traumatic lesions of the basal layer of the endometrium. Its real prevalence is difficult to assess because of the existence of pauci or asymptomatic forms. In Cameroon it has been estimated at $1 \%$ in a pioneering study on the subject. ${ }^{1}$

The clinical presentation is dominated by menstrual disorders such secondary amenorrhea or hypomenorrhea, and infertility. 
The diagnostic presumption is made on ultrasound, hysterosonography and hysterosalpingography. However, hysteroscopy remains the most appropriate diagnostic and therapeutic means for uterine synechiae.

Hysteroscopy did not experience the same development as laparoscopy in Cameroon. As a result, a presumptive diagnosis of uterine synechia has remained a source of stress for the practitioner, with sometimes less accepted treatments nowadays, such as dilation with Hegar candles or laparotomy.

Today in Cameroon, CHRACERH which is a center of excellence in endoscopic surgery, has a good technical platform for the diagnosis and treatment of uterine synechiae which until now is not very common in the Cameroonian and even African scientific setting. Our study aimed to fill this gap and to evaluate the diagnostic, therapeutic and prognostic aspects of hysteroscopic synechia treated in CHRACERH.

\section{METHODS}

We conducted a descriptive cross-sectional study with an analytical component. Data collection was retrolective. It was performed at the Hospital Center for Research and Application in Endoscopic Surgery and Human Reproduction (CHRACERH) in Yaoundé, Cameroon. It is a hospital facility whose objective is to conduct research in endoscopy and human reproduction. It also provides high-level health care in gynecology and obstetrics. For gynecological surgery, it has a capacity of 30 hospital beds and 4 operating rooms. Endoscopic surgery, which accounts for nearly $45 \%$ of all gynecological surgery, is performed by gynecologic/obstetric surgeons assisted by anesthetist/intensive care physicians. The imaging area is equipped with ultrasound, mammography and hysterosalpingography equipment. The medical activity is practiced by specialists in radiology and medical imaging and some gynecologists specializing in gynecological and obstetrical ultrasound.

This study covered the period from January 1st 2015 to July 31 st 2017 , for a duration of 2 years 7 months. All the patients seen in gynecological consultation or followed in our center constituted our source population.

The target population consisted of women in whom uterine synechiae were found during hysteroscopy. Ethical considerations were respected on the anonymity and confidentiality of the data collected. The size of the sample was calculated taking into account the prevalence of uterine synechia of $1 \%$ found by Tchounzou. ${ }^{1}$ This allowed to estimate a minimum sample size of 15 patients. We included all patients with uterine synechia managed by hysteroscopy at CHRACERH and who had a complete medical record and who agreed to participate in the study. Those who were not treated by hysteroscopy or had incomplete medical records, or refused to participate in the study, were not included.

The data were collected on a validated and pre-tested data sheet, using hospital records, anesthesia charts and patient reports. Some information were completed by the patients during an interview after recall. For those who could not honor their appointment, the information was collected by telephone interview. Parameters of interest were sociodemographic, gynecological and obstetric past history and extra-gynecological antecedents in search of etiological factors of uterine synechiae. The duration of exposure was defined as the time between the date of occurrence of this factor and the date of diagnosis, expressed in months. We also looked for functional signs including menstrual disorders and the type of infertility. Data from the gynecological examination and the results of additional imaging examinations: pelvic ultrasound, hysterosonography, hysterosalpingography were collected. The report of the diagnostic hysteroscopy allowed to describe the type of synechia (mucous, fibrous, muscular), the extent of the synechia (in 1/3, 2/3 or $3 / 3$ of the uterine cavity). Localization and visualization or not of tubal ostia was noted. Finally, the synechiae were classified according to the classification of the American Fertility Society. ${ }^{2}$

The correlation between the paraclinical imaging examinations and diagnostic hysteroscopy was assessed by the sensitivity test and the positive predictive value. The operative data we searched for were: the type of instrument used for the section of the synechiae (hysteroscopic scissors, tip of the hysteroscope, resectoscope), the ultrasound guidance of the hysteroscopic proccedure. The per and postoperative complications, the type of complication. Treatment results anatomically (total or partial recovery of the uterine cavity or failure if it is impossible to restore even partially the uterine cavity during a cure). Functionally, the normalization of disorders of the menstrual cycle or their improvement and a possible recovery of fertility found or not.The anatomical results were judged by the total or partial restitution of a morphologically normal uterine cavity. The failure of the treatment was defined by the impossibility of restoring even partially the uterine cavity after having begun the hysteroscopic procedure. The functional results have been, on the one hand, judged by the normalization of the disorders of the menstrual cycle or their improvement, and on the other hand, by a recovered fertility or not. A good result on menstrual disorders, associated normalization and improvement of the disorder. Failure was the postoperative persistence of the disorder diagnosed before the operation.

\section{Statistical analysis}

The data was recorded and analyzed using Microsoft Excel 2016 and SPSS 24.0 statistics software. Qualitative data were expressed as absolute or relative frequency and quantitative data as mean \pm standard deviation. They have 
been represented in the form of figures or tables. The Chi square test (X2) was used for the comparison of qualitative variables and the Fisher $(\mathrm{F})$ test for analysis of variance (ANOVA). The correlation was evaluated by the Spearman correlation test. P-value less than 0.05 was considered as statistically significant.

\section{RESULTS}

During the period under review, there were a total of 30 cases of uterine synechiae managed by hysteroscopy on 207 hysteroscopic procedures. Thus, hysteroscopic management of uterine synechia constituted $14.5 \%$ of all hysteroscopic procedure.

The mean age of the patients was $40.10 \pm 6$ years with extremes ranging from 28 years to 55 years. $53.30 \%$ $(16 / 30)$ of patients were under age 40 .

Table 1: Etiological factors of uterine synechia.

\begin{tabular}{|c|c|c|}
\hline History of uterine trauma & No. of case & Percentage \\
\hline \multicolumn{3}{|l|}{ Trauma on pregnant uterus } \\
\hline Postabortum curettage & 16 & 53.33 \\
\hline Post abortum aspiration & 04 & 13.33 \\
\hline Caesarean section & 05 & 16.67 \\
\hline Uterine revision & 02 & 6.67 \\
\hline Postpartum endometritis & 02 & 6.67 \\
\hline Postabortum endometritis & 01 & 3.33 \\
\hline \multicolumn{3}{|c|}{ Trauma on non-pregnant uterus } \\
\hline Intrauterine surgery & 20 & 66.67 \\
\hline Intrauterine device & 07 & 23.33 \\
\hline Genital tuberculosis & 01 & 3.33 \\
\hline Unknown Etiology & 02 & 6.67 \\
\hline Total & 30 & 100 \\
\hline
\end{tabular}

The mean gestity was $2.43 \pm 2.36$ with extremes of 0 to 12 . Four patients $(13.3 \%)$ were nulligested. The average parity was $0.47 \pm 0.57$ with extremes of 0 to 2 . Seventeen patients $(56.70 \%)$ were nulliparous.

Several etiological factors were identified from the gynecological obstetric history of the patients and are summarized in Table 1. The Table 1 shows that among the etiological factors investigated, post-abortion curettage was the most common etiological factor found in the pregnant uterus with $53.33 \%$ of cases $(16 / 30)$.

In non-pregnant uterus, intrauterine surgery was the largest provider of synechia with $66.67 \%$ cases (20/30). Myomectomy was performed by laparotomy in 11 cases $(36.67 \%)$. Operative hysteroscopy was perfomed in 9 patients $(30 \%)$ and hysteroscopic myomectomy was realized in $6 / 9$ patients $(66.67 \%)$.

All patients had infertility of which $86.70 \%$ (26/30) was secondary infertility. This infertility was associated with menstrual disorder in $83.33 \%(25 / 30)$.
Table 2: Correlation between imaging and diagnostic hysteroscopy.

\begin{tabular}{|llll|}
\hline $\begin{array}{l}\text { Paraclinical } \\
\text { imaging }\end{array}$ & No. & $\begin{array}{l}\text { Positive } \\
\text { results }\end{array}$ & $\begin{array}{l}\text { Sensitivity } \\
(\%)\end{array}$ \\
\hline $\begin{array}{l}\text { Pelvic } \\
\text { ultrasound }\end{array}$ & 24 & 6 & 25 \\
\hline Hysterosonography & 11 & 4 & 36.36 \\
\hline Hysterosalpingography & 17 & 15 & 88.23 \\
\hline
\end{tabular}

The paraclinical imaging examinations allowed to compare the results with the hysteroscopy in the diagnosis of the uterine synechies (Table 2).

Table 2 shows that ultrasound and hysterosonography were less sensitive than hysterosalpingography. However, the sensitivity of the hysterosalpingogram is less for the localization of the synechiae: it was about $26.67 \%$ (4 good localizations on 15 mentioned localizations).

The diagnostic hysteroscopy showed that $63.30 \%$ (19/30) of synechias were fibrous, $30 \%$ (9/30) were mucous and $6.7 \%(2 / 30)$ muscular synechias. There is a strong positive correlation between the type of hysteroscopic view of synechia and the duration of exposure to the etiological factor (Spearman correlation coefficient $=0.629 ; \mathrm{p}=0.000$ ).

According to the American Fertility Society (AFS), Most uterine synechiae were classified as stage II (56.7\%), followed by stage I (30.0\%) and Stage III (13.30\%).

Table 3: Anatomical results according to the AFS classification.

\begin{tabular}{|lllll|} 
Stages & Failure & $\begin{array}{l}\text { Partial } \\
\text { restitution }\end{array}$ & $\begin{array}{l}\text { Complete } \\
\text { restitution }\end{array}$ & Total \\
\hline I & 0 & 0 & 9 & 9 \\
\hline II & 1 & 8 & 8 & 17 \\
\hline III & 2 & 0 & 2 & 4 \\
\hline Total & 3 & 8 & 19 & 30 \\
\hline
\end{tabular}

After hysteroscopic treatment, we observed $63.30 \%$ cases of complete anatomic restitution and $26.70 \%$ cases of partial restitution. In 3 patients, no surgical procedure was possible. These were classified as treatment failure. Table 3 shows anatomical results according to the AFS classification.

There is a moderate and negative correlation between stage of synechia and anatomic outcome (spearman correlation coefficient $=-0.477 ; \mathrm{p}=0.008$ ).

Results on menstrual disorders according to the AFS classification showed that good results on menstrual disorder was found in $83.30 \%$ of stage I, $80 \%$ of stage II and $33.30 \%$ of stage III. Table 1-4 summarizes results on menstrual disorders. 
Table 4: Results on menstrual disorder according to the AFS classification.

\begin{tabular}{|lllll|}
\hline Stages & Failure & Improvement & Normalization & Total \\
\hline I & 1 & 2 & 3 & 6 \\
& $(16.70 \%)$ & $(33.30 \%)$ & $(50 \%)$ & 6 \\
II & 3 & 7 & 5 & 15 \\
& $(20 \%)$ & $(46.70 \%)$ & $(33.30 \%)$ & \\
III & 2 & 0 & 1 & 3 \\
\hline Total & $(66.70 \%)$ & $(0 \%)$ & $(33.30 \%)$ & 3 \\
\hline
\end{tabular}

The difference between different stages of severity was not statistically significant and similarly, there was no correlation between stage of synechia and outcome on menstrual disorders (spearman correlation coefficient $=-$ $0.221 ; \mathrm{p}=0.300)$.

\section{DISCUSSION}

Intrauterine adhesions or uterine synechiae are defined as coalescence of the uterine walls between them, whatever their location from the external orifice of the cervix. They constitute an infrequent pathology in our daily practice, representing $14.5 \%$ of indications of operative hysteroscopies at CHRACERH. The universal incidence of uterine synechiae is not really known and varies in the literature according to the history and the symptomatology of the patients. ${ }^{3}$ It is about $2.75 \%$ in asymptomatic women without risk factors, between $3.7 \%$ and $23.4 \%$ in case of postabortum or postpartum curettage, from $1.7 \%$ to $9 \%$ when there is secondary amenorrhea. ${ }^{4,5}$ In our series, reporting the number of cases to the number of gynecological consultations, the incidence is $0.25 \%$. We cannot compare this incidence to the above cited literature because of the different methodologies used to conduct these studies. An incidence of $1 \%$ was found by Tchounzou et al in a regional hospital in Cameroon where there are fewer patients compared to CHRACERH, which serves the entire sub-region of Central Africa and more. ${ }^{1}$

In our series, the mean age of the patients was 40.1 years, higher than that reported in the literature..$^{6-9}$ This difference can be explained by the fact that in Africa, the specialist is consulted as a last resort, often after several years of searching for the solution to traditional practitioners or to prayers.

Infertility was the main reason for consultation in all patients. The majority $(87 \%)$ were secondary infertility. This result is comparable to that reported in the literature. ${ }^{8-11}$ Some synechiae may be the real cause of infertility (cervico-isthmic, total or complex synechia), in other cases the causality is less clear and a complete health check of the couple is essential before any therapy.

Uterine synechiae are most often of post-traumatic origin, by destruction of the basal layer of the endometrium. They are thus particularly important in gynecological practice where endo-uterine maneuvers are most often performed. The maneuvers on gravid uterus, remain the main etiology of uterine synechiae: the first cause being curettage and / or aspiration after spontaneous or induced abortion. In our series, $66.66 \%$ of patients had a past history of abortion, mainly miscarriages, managed by curettage or aspiration (Table 1). Our results corroborate with those of the literature which shows that, for more than 30 years, the cause of synechia remains the curettage on gravid uterus in $60.8 \%$ to $97 \%$ of cases. ${ }^{8,10-15}$

Some authors, like Darai, think that synechia is more frequent after curettage with the soft curette than after endo-uterine aspiration. ${ }^{4}$ Present results seem to confirm this hypothesis, especially in post-abortion. The practice of postpartum curettage is not widespread in our country, it is replaced by uterine revision $(6.67 \%)$ or artificial delivery $(3.33 \%)$, gestures that appear little traumatic for the uterine cavity. The history of caesarean section was found in $16.67 \%$ of cases. Uterine revision during caesarean section and placental retention are most often involved in the occurrence of synechiae. The extramucous suturing of the hysterotomy, may help to reduce the incidence. ${ }^{4}$ Among non-pregnant uterine surgeries, myomectomy appears to be an important etiological factor irrespective of the surgical approach. The responsibility of myomectomy by laparotomy in the occurrence of synechiae can only be evoked when there is a break in the uterine cavity and preferentially for submucosal myomas. In the literature, the postmyomectomy synechiae rate varies from 1.2 to 4.2 , if performed by laparotomy. ${ }^{4,8,10}$ In our series, we found a history of myomectomy by laparotomy in $36.67 \%$ of cases. The higher frequency of myomas in the black race may explain this difference. Hysteroscopic myomectomy is responsible for synechiae especially when it concerns a polymyomectomy. Hysteroscopic surgery with endouterine resection has now become an important cause of synechia: $8.5 \%$ of cases in Blanc et al's series, and 30\% of the patients in our series had a history of operative hysteroscopy of which more than half $(66.67 \%)$ of the cases were myomectomy. ${ }^{16}$ These synechiae are usually of good prognosis if they are diagnosed and treated during early second hysteroscopic look within two to three months. But they are sometimes extremely difficult to treat when they are extensive, complex or muscular. In some situations, no endo-uterine manipulation is found. The existence of post-infectious uterine synechiae may in this case be very suggestive of the infectious origin. Some authors such as Rabau et al believe that infection, whether acute, subacute, chronic, or even asymptomatic, remains the primum movens of uterine synechiae. ${ }^{17}$ This theory is based on three findings reported by these authors: the existence of intra-tubal adhesions after postcurettage salpingitis, the histological exploration of synechiae is frequently in favor of subacute or chronic endometritis and the existence on anamnesis of a high frequency of symptoms (leukorrhea, pelvic pain) suggestive of infection, in patients who underwent uterine curettage and who are carriers of synechiae. However, for 
other authors, the role of endometritis due to common germs in the genesis of uterine synechia remains controversial. $^{4,18}$ In a series of 171 post-caesarean women, Polishuk et al, found no significant difference in uterine synechiae rates between patients who had clinically proven post-cesarean endometritis by bacteriological analysis and those who had no signs of infection after Caesarean section. ${ }^{18}$ In present series, endometritis was reported in 3 cases $(10 \%)$ of which 2 in postpartum and one in postabortum. Chronic inflammation of the endometrium may also be suggestive of genital tuberculosis that is endemic in our country. One of our patients $(3.3 \%)$ had genital tuberculosis.

Symptomatically, all our patients had infertility. This is consistent with the literature where in all the series, infertility mainly secondary, was the main reason for consultation. ${ }^{8-11}$ Secondary infertility was present in $87 \%$ of cases in our series. In case of uterine synechia, infertility seems to be multifactorial. It is linked on the one hand, to the presence of mechanical obstacle: the occlusion of the tubal ostia, and / or the complete stenosis of the uterine cavity, or of the cervical canal, can impede the migration of the spermatozoa and the implantation of the egg. ${ }^{19}$ On the other hand, the alteration of the adjacent endometrium by lack of hormonal receptivity as well as its vascular degradation prevents the proper course of implantation and placentation. In uterine synechiae, menstrual disorders dominated by amenorrhea, oligomenorrhea, and hypomenorrhea are common, and usually after infertility, constitute the second circumstance of discovery of synechia. ${ }^{8-10}$ Present series confirms this hypothesis with a menstrual disorder in $86.70 \%$ of cases, including $26.70 \%$ of cases of amenorrhea, $26.70 \%$ of cases of oligomenorrhea and $10 \%$ of cases of hypomenorrhea. Uterine synechiae rarely manifest as dysmenorrhea except in case of cervical synechiae. Dysmenorrhea is often due to an associated pathology, such as endometriosis resulting from increased menstrual tubal reflux secondary to obstruction. ${ }^{20}$ Menorrhagia and metrorrhagia are not often related to the presence of synechia, but rather to the associated endometrial lesions. A normal cycle does not exclude the presence of synechiae, which are most often partial. $^{21}$ In current series, $13.30 \%$ of patients had no menstrual disorder. Clinical examination, most often noncontributory, makes one suspect the diagnosis of synechia only with the impossibility of catheterizing the cervix with a hysterometer or with a fine probe. However, this sign is only found for complete or near-complete cervicoisthmic synechiae. ${ }^{4}$

Endovaginal pelvic ultrasound can be of great help in distinguishing cervico-isthmic synechiae from the total synechia of the uterine cavity. Typically, the synechiae will be in the form of dense intra-cavitary echoes, and in the case of cervico-isthmic synechiae, the emptiness line is normal, and we can appreciate the quality of the endometrium as well as the thickness of the endometrium. In general, however, the sensitivity and specificity of ultrasound in the diagnosis of uterine synechiae remains limited. ${ }^{22,23}$ We found a sensitivity of $25 \%$. The interest of 3D ultrasound has been mentioned by these authors. Hysterosonography, which combines transvaginal ultrasound and isotonic saline injection, has been shown to be as accurate as hysterosalpingography and superior to transvaginal ultrasound in the detection of uterine synechiae. ${ }^{22}$ But in our series, we found a sensitivity of hysterosonography at $36.36 \%$. Indeed, some synechiae, when they are partial and thin, can be lifted during cannulation. Diagnostic hysteroscopy is the key examination in the diagnosis and assessment of synechiae. It can sometimes correct some false positives of hysterosalpingography (HSG). The HSG nevertheless keeps its place in the diagnosis of uterine synechiae especially as it allows the diagnosis of associated lesions, especially tubal lesions: it is therefore the first-line examination if infertility is the reason for consultation. ${ }^{24}$ In $56.70 \%(17 / 30)$ of cases, our patients performed this test. The hysterosalpingographic image of a uterine synechia corresponds to a subtraction image-shaped, irregularly shaped, linear or angular gap with clear or sharp edges. This gap is constant on all shots that must be read during all phases (filling, repletion, evacuation, profile) to avoid confusion with other lesions (polyps, fibroids, septum, hyperplasia, air bubble). In our series, we found a sensitivity of $88.23 \%$ for the diagnosis of uterine synechiae to HSG, confirmed by hysteroscopy. The confrontation of HSG and hysteroscopy shows a good correlation in the diagnosis of uterine synechiae in general with a sensitivity that varies from $52.6 \%$ to $90 \%$ in the literature. ${ }^{8,9,12,15}$ On the other hand, for the localization of synechia, we found a low sensitivity at $26.67 \%$, confirming those of Daaloul, which had a sensitivity of $40 \% .^{8}$ Nowadays, hysteroscopy is the gold standard for the diagnosis and treatment of uterine synechiae. It specifies the type of synechia (mucous, fibrous or muscular), the localization, the extent and appreciates the associated intra-cavitary lesions and the appearance of the remaining endometrium. This makes it possible to formulate a prognosis. There are several classifications of synechiae that help predict the prognosis and outcome of treatment. However, none of them is unanimous. We adopted the American Fertility Society (AFS) classification: $56.70 \%$ of patients were classified as stage II. ${ }^{2}$ This classification has the merit of correlating the menstrual history and the severity of the uterine synechias, although it does not correlate the severity of the synechies and their sites. ${ }^{25}$ March's classification, although simple and applicable, does not provide information on the prognosis of uterine synechiae. ${ }^{26}$

The goal of the treatment of uterine synechiae is to restore the normal anatomy of the uterine cavity, prevent re-walling, help regenerate the endometrium and remotely control anatomical findings. Satisfactory anatomical results vary in the literature and whether one is at the first hysteroscopy or second or higher. The rate of anatomical success during a first session varies from 
$63.8 \%$ to $97.4 \%$, and from $66 \%$ to $94.3 \%$ in a second look. ${ }^{9-12,15}$ Some authors found a rate of $96.7 \%$ in a third session. ${ }^{10}$ After hysteroscopic treatment, we observed $63.30 \%$ cases of complete anatomic restitution and $26.70 \%$ cases of partial restitution. However, it is more difficult to obtain a good result when the synechia is initially classified as severe. ${ }^{27}$ Present study confirms this finding: There was a moderate and negative correlation between stage of synechia and anatomic outcome (spearman correlation coefficient $=-0.477 ; \mathrm{p}=0.008$ ). In $70 \%$ of cases, synechiae were rated at least moderate in our series: This may explain our lower overall success rate than in the literature.

The results on menstrual disorders seem to be good. Of the 24 patients who had a menstrual disorder, there was a disappearance of symptomatology in 9 patients $(37.5 \%)$ and a significant improvement in 9 patients $(37.5 \%)$, thus $75 \%$ of good results. Our results are the same to those found in the literature with a percentage of good results on menstrual disorders ranging from $75 \%$ to $88.46 \%{ }^{9}$ 11,28 Roge et al found that the results on menstrual disorders are all the better as synechia is less severe. ${ }^{11}$ Present results confirm this observation. In fact, $83 \%$ of patients classified as stage I, $80 \%$ classified as stage II and only $33.3 \%$ as stage III had good results, although overall, the result did not vary significantly between groups $(\mathrm{p}=0.300)$.

\section{CONCLUSION}

Hysteroscopic management of uterine synechia represents $14.5 \%$ of hysteroscopies and $0.25 \%$ of all consultations. The most affected women are pauciparous, and often under 40 years old. They often present with menstrual disorders, in the context of secondary infertility, with in their antecedents a history of curettage or myomectomy with endometrial break-in. Hysterosalpingography demonstrated good sensitivity estimated to be $88.23 \%$ better than endovaginal ultrasound and hysterosonography. Hysteroscopic management of uterine synechiae has good and significant anatomic results, especially when synechiae is initially classified as mild to moderate. But these results were not significant for restitution of a normal menstrual cycle. Prevention is essentially based on the prevention of all the risk factors involved, particularly curettage, which is the most common etiology of uterine synechiae.

Funding: No funding sources

Conflict of interest: None declared

Ethical approval: The study was approved by the Institutional Ethics Committee

\section{REFERENCES}

1. Tchounzou R, Ngono M, Moifo B, Mbu RE. Treatment of uterine synechiae in the absence of hysteroscopy in semi-urban areas in Cameroon. Med Sante Trop. 2014;24:263-5.
2. Society TAF. The American Fertility Society classification of adnescal adhesions, distal tubal occlusion secondary to tubal ligatio, tubal pregnancies, Mullerian anomalies and intrauterine adhesions. Fertil Steril. 1992;49:944-55.

3. Efetie ER. Reproductive outcome following treatment of intrauterine adhesions in Abuja, Nigeria. Niger J Clin Pract. 2006;9:164-8.

4. Daraï E, Deval B, Benifla JL, Guglielmina JN, Sitbon D, Filippini F, et al. Uterine synechias. EMC Gynecology. 1996;155-A-30:1-11.

5. Bergman P, Wehlin L. Post-traumatic intra-uterine synechiae. Acta Radiol. 1958;49:453-63.

6. McComb PF, Wagner BL. Simplified therapy for Asherman's syndrome. Fertil Steril. 1997;68:104750.

7. Protopapas A, Shushan A, Magos A. Myometrial scoring: a new technique for the management of severe Asherman's syndrome. Fertil Steril. 1998;69(5):860-4.

8. Daaloul W, Ouerdiane N, Masmoudi A, Ben HS, Bouguerra B, Sfar R. Epidemiological profile, etiological diagnosis and prognosis of uterine synechias: report of 86 cases. Tunis Med. 2012;90(4):306-1.

9. Chanoufi MB, Chelli D, Ghaffari C. Uterine synechiae: results after hysteroscopic treatment in 127 cases. Gynecologist's Letter. 2006;30:9-14.

10. Kdous M, Hachicha R, Zhiou F, Ferchiou M, Chaker A, Meriah S. Fertility after hysteroscopic treatment of intra-uterine adhesions. Gynecol Obstet Fertil. 2003;31:422-8.

11. Roge P, D'ercole C, Cravello L, Boubli L, Blanc B. Hysteroscopic treatement of uterine synechias. A report of 102 case. J Gynecol Obstet Biol Reprod. 1996;25(1):33-40.

12. Hamou J, Cittadini E, Perino A. Diagnosis and management of intrauterine adhesions by microhysteroscopy. Acta Eur Fertil. 1983;14:117-23.

13. Schenker JG, Margalioth EJ. Intrauterine adhesions: an updated appraisal. Fertil Steril. 1982;37:593-610.

14. Valle RF, Sciarra JJ. Intrauterine adhesions: hysteroscopic diagnosis, classification, treatment, and reproductive outcome. Am J Obstet Gynecol. 1988;158(6Pt1):1459-70.

15. Lancet M, Kessler I. Treatment of Asherman's syndrome with hysteroscopy and concomitant hysterography. J Gynecol Obstet Biol Reprod. 1986;15:464-6.

16. Blanc B, Boubli L. Uterine endoscopy. Paris Pradel editions; 1996.

17. Rabau E, David A. Intrauterine adhesions: etiology, prevention, and treatment. Obstet Gynecol. 1963;22:626-9.

18. Polishuk WZ, Anteby SO, Weinstein D. Puerperal endometritis and intrauterine adhesions. Int Surg. 1975;60:418-20.

19. Yu D, Wong YM, Cheong Y, Xia E, Li TC. Asherman syndrome: one century later. Fertil Steril. 2008;89(4):759-79. 
20. Bricou A, Demaria F, Boquet B, Jouannic JM, Benifla JL. Uterine synechias. EMC-Gynecology. 158-A-10 2009:1-13.

21. Taylor PJ, Cumming DC, Hill PJ. Significance of intrauterine adhesions detected hysteroscopically in eumenorrheic infertile women and the role of antecedent curettage in their formation. Am J Obstet Gynecol. 1981;139(3):239-242.

22. Salle B, Gaucherand P, De Saint Hilaire P, Rudigoz RC. Transvaginal sonohysterographic evaluation of intrauterine adhesions. J Clin Ultrasound. 1999;27:131-4.

23. Sylvestre C, Child TJ, Tulandi T, Tan SL. A prospective study to evaluate the efficacy of two- and three-dimensional sonohysterography in women with intrauterine lesions. Fertil Steril. 2003;79:1222-5.

24. Eng CW, Tang PH, Ong CL. Hysterosalpingography: current applications. Singapore Med J. 2007;48:36874.

25. Al-Inany H. Intrauterine adhesions. An update. Acta Obstet Gynecol Scand. 2001;80:986-93.
26. March CM, Israel R. Intrauterine adhesions secondary to elective abortion. Hysteroscopic diagnosis and management. Obstet Gynecol. 1976;48:422-4.

27. Chen FP, Soong YK, Hui YL. Successful treatment of severe uterine synechiae with transcervical resectoscopy combined with laminaria tent. Hum Reprod. 1997;12:943-7.

28. Schenker JG. Etiology of and therapeutic approach to synechia uteri. Eur J Obstet Gynecol Reprod Biol. 1996;65(1):109-113.

Cite this article as: Belinga E, Tompeen I, Ndoua CCN, Ntsama JM, Mendibi S, Kasia JM. Diagnostic, therapeutic and prognostic aspects of uterine synechiae managed by hysteroscopy in the gyanecological endoscopic surgery and human reproductive teaching hospital. Int J Reprod Contracept Obstet Gynecol 2018;7:56-62. 